\title{
Streptococcus oralis
}

National Cancer Institute

\section{Source}

National Cancer Institute. Streptococcus oralis. NCI Thesaurus. Code C86799.

A species of facultatively anaerobic, Gram positive, cocci shaped bacteria in the phylum Firmicutes. This species is alpha hemolytic, urease and catalase negative, sialidase and alpha glucosidase positive and does not hydrolyze esculin. It can ferment ribose, raffinose, and trehalose but not inulin, cellobiose, mannitol, or sorbitol. S. oralis is found in the human oral cavity and respiratory tract and can be a pathogen that causes dental caries, gum disease, and endocarditis following oral trauma. 